\title{
Adjacency Effects in Developmental Correlations Among Tooth Organs in Human Fetuses
}

\author{
ALPHONSE R. BURDI,* JEROME SUPERSTINE, ${ }^{* *}$ and WILLIAM BABLER***
}

Departments of Anatomy* and Orthodontics,** The University of Michigan, Ann Arbor, Michigan 48109 and Department of Anatomy, *** University of Virginia, Charlottesville, Virginia 22904

This study explores whether the "distance gradient" model shown for embryonic development and postnatal tooth growth is also characteristic of the fetal period. Histologic data and ratings from 26 human fetuses show that, unlike the embryonic and postnatal periods, changes occurring in two tooth germs are significantly alike, regardless of the number of intervening teeth.

J Dent Res 60(2):171-173, February 1981

\section{Introduction.}

Studies of human postnatal crown size development have shown a "distance gradient" in which increases in crown size of teeth closer together within the jaw quadrant are highly correlated, regardless of morphologic tooth type. Adjacent teeth exhibit systematically higher correlations in crown size than two teeth separated by one or more teeth..$^{1-3}$ With the extension of this postnatal "distance gradient" model to the beginning stages of tooth development from six to ten intrauterine wk, histologic changes in early bud- through early bell-staged tooth germs which were adjacent within the area showed the highest developmental similarities. Accordingly, tooth germs separated by one tooth germ showed lower correlations, while tooth germs separated by two intervening tooth germs showed still lower correlations. $^{4}$

Based on these background observations of a "distance gradient" in postnatal changes in deciduous tooth size and in the much earlier morphologic stages of intrauterine tooth formation, this present study extends this concept of a "distance gradient" in tooth morphogenesis into the intervening and yet unexplored fetal period.

Received for publication January 28, 1980 Accepted for publication May 8, 1980

This investigation was supported, in part, by U.S.P.H.S. Grants DE 03443 and DE 03610 from the National Institute of Dental Research.

\section{Materials and methods.}

Twenty-six human fetuses, representing weeks ten through 36 of intrauterine development (i.e., 75-345 mm crown-rump length), were randomly chosen for study. Each specimen was considered "normal," or typical-for-age, based on gross physical examination at time of operating room delivery and because no signs of abnormality were indicated in either the clinical or familial histories accompanying each specimen. Upper and lower jaws from each specimen were serially sectioned at 7 to 10 micra and stained with Mallory trichrome connective tissue stain for subsequent light microscopy. Sections through each available deciduous tooth germ (di1-di2-dc-dm1-dm2) were rated for their progressive morphologic or histologic changes seen through the tenthto thirty-six th-week period of study. Fifteen histologic stages were used to identify the development or body length of a given tooth germ at a particular time. This previously reported tooth germ staging scheme ${ }^{5}$ covered stages and intermediate levels of development beginning with the early appearance of the dental lamina as a primary epithelial invagination at the margins of the oral cavity. Following the various stages of bud and cap formation, which included modifications in size and shape of the tooth germs, the last of the 15 histologic stages focused on the appositional phases of tooth formation, featuring the deposition of the enamel and dentin matrices. Although most readings were done by a single observer (JS), multi-observer readings were made to ascertain intra- and inter-observer reliability in tooth germ staging. Reliability coefficients were in excess of 0.98 , and interobserver staging errors were of the order of \pm 0.05 for larger specimens, and \pm 0.01 for the smaller specimens of the series.

Similar to increases in fetal body stature (i.e., crown-rump length or $\mathrm{CRL}$ ) from 75 $345 \mathrm{~mm}$ in this cross-sectional population 
of human fetuses, data from the various stages of tooth germ development were also treated as continuous variables. Based on tooth-by-tooth readings from each of the 26 fetuses, correlation matrices between tooth germs were calculated using both Pearson product-moment $(r)$ and partial correlation $\left(\mathrm{r}_{12.3}\right)$ values. While the usefulness of $r$ values in growth studies is generally known for showing the relationship between the degree of development in a given embryonic part and body size, the partial correlation $\left(\mathrm{r}_{12 \cdot 3}\right)$ was used in this study as an appropriate procedure to hold the fetal crown-rump length variate constant while testing the correlation between developmental stages among tooth germs. ${ }^{6-7}$ Thus, partial correlations, in effect, eliminate spuriously large values of $r$ which can result from the fetal body size factor alone. In this study, inter-tooth partial correlations $\left(r_{12.3}\right)$ will be referred to as $r_{C R L}$, since it is the crown-rump length which is being held constant.

\section{Results.}

As shown in Figs. 1 and 2, differences do occur in the absolute correlation values $(r$ and $r_{C R L}$ ). Using pooled data from right and left jaw quadrants, Fig. 1 shows that, while tooth germs separated by three other tooth germs (i.e., dil-dm2) show reduced correlation values, the histologic maturation of all tooth germs during the ten- to 36-week period is significantly similar (at 0.01 level) regardless of morphologic tooth type (i.e., incisor, cuspid, molar) and the number of tooth germs between any pair of tooth germs being compared. When compared with partial correlation $\left(\mathrm{r}_{\mathrm{CRL}}\right)$ statistics, partial correlation values (although lower than the Pearson product-moment $r$ values shown in parentheses) also confirm the observation that no significant difference can be found in the histologic maturation of the adjacent or widely separated tooth germs during the ten- to 36-week fetal period when crownrump length is held constant. This developmental similarity among each of the fetal tooth germs is demonstrated in Fig. 2, which presents $r$ and partial correlation values $\left(r_{C R L}\right)$ in the form of one-away, two-away, three-away models to show that the number of intervening tooth germs between any two tooth germs being compared for maturational advancement does not affect the existing significant and positive correlations between the two tooth germs, even though tooth-tooth correlations involving $\mathrm{dm} 2$ generally show lower correlation values.

\section{DECIOUOUS TEETH}

Mandibular Moxillory
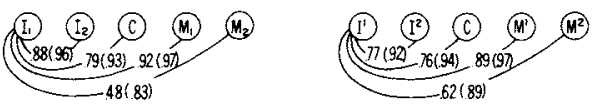

(i)

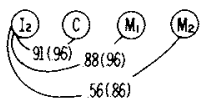

(I)

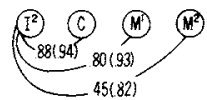

(11)
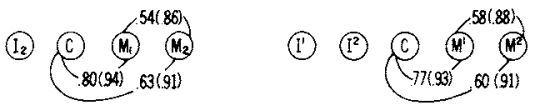

Fig. 1 - Multiple correlations involving 15 histologic stages of individual deciduous tooth germ formation of pooled maxillary and mandibular jaw quadrants using raw order correlation $r$ (in parentheses) and partial correlation values, i.e., $\mathrm{r}$ CRL.
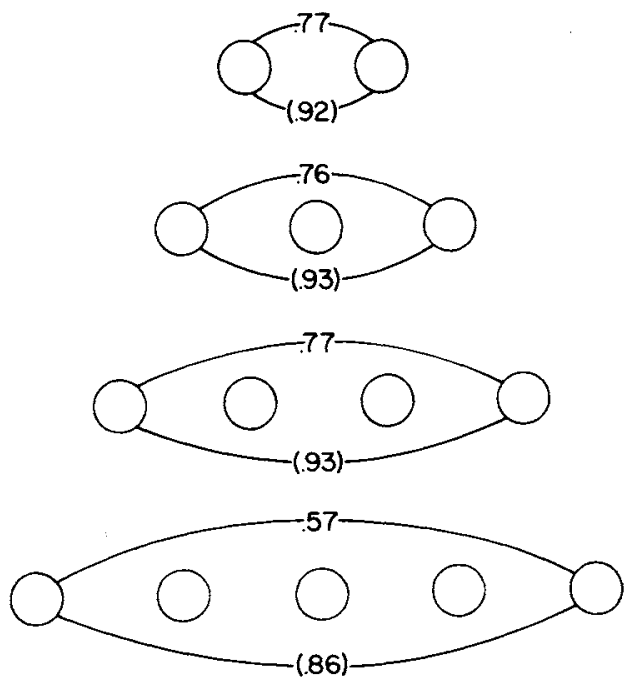

Fig. 2 - Distance or adjacency models based on pooled correlation values of all tooth germs grouped according to their separation by one, two, or three tooth germs regardless of morphological tooth type. Raw order $(r)$ and partial correlation, i.e., rCRL, values are keyed as in Fig. 1. 


\section{Discussion.}

This study shows that, while a "distance gradient" model has been reported for postnatal crown sizes and for the earliest stages of human tooth morphogenesis at six to ten intrauterine $\mathrm{wk}^{4}{ }^{4}$ significant differences in histologic development between two tooth germs are not demonstrable in the fetal period using a continuation of the same tooth scoring scheme used in the earlier embryonic period. These analyses of ten- to 36-week-old human fetuses show that the concept of a "distance gradient" in tooth development is time-specific and not characteristic of the entire pre- and postnatal continuum of development. Instead, the gradient first appears during the embryonic period, is minimal or absent during the fetal period, and reappears during the postnatal period. The absence in the fetal period of the "distance gradient" seen earlier in the embryologic period is coincidental with and may reflect the progressive calcification of the developing tooth germs. ${ }^{8}$

\section{Conclusions.}

This study recognized earlier literature reports showing that the developmental and crown size correlations among deciduous teeth will decrease in accordance with the number of intervening teeth between the two teeth being compared, i.e., the "distance gradient" model. Based on statistical treatment of cross-sectional data derived from a human fetus population, this study shows that, while a "distance gradient" model is discernible for the young human prenate six- to ten-weeks-old and for changes in tooth crown size in the child, the "distance gradient" is not significantly discernible during the much longer fetal period (ten to $36 \mathrm{wk}$ ) in those parameters of tooth morphogenesis which were monitored.

\section{REFERENCES}

1. GARN, S.M.; LEWIS, A.B.; and KEREWSKY, R.S.: Relationships of the Mesial and Distal Teeth, $J$ Dent Res 44:350-354, 1965.

2. GARN, S.M.; LEWIS, A.B.; and KEREWSKY, R.S.: Shape Similarities Throughout the Dentition, $J$ Dent Res 46:1481, 1967.

3. GARN, S.M.; ROHMANN, C.G.; BLUMENTHAL, T.; and SILVERMAN, F.N.: Ossification Communalities of the Hand and Other Body Parts, Amer J Phys Anthropol 27:7583, 1967.

4. GARN, S.M.; BURDI, A.R.; and NAGY, J.M.: Distance Gradient in Prenatal Dental Development, J Dent Res 50:785, 1971.

5. GARN, S.M. and BURDI, A.R.: Prenatal Ordering and Postnatal Sequence in Dental Development, $J$ Dent Res 50:1407-1414, 1971.

6. SIMPSON, G.G.; ROE, A.; and LEWONTIN, R.C.: Quantitative Zoology, New York: Harcourt, Brace and Company, 1960.

7. BURDI, A.R. and SUPERSTINE, J.: Developmental Correlations of the Deciduous and Permanent Teeth During the Human Fetal Period, J Dent Res 56:1468, 1977.

8. KRAUS, B.: Calcification of the Human Dentition, JADA 59:1128-1136, 1959. 